\title{
The effect of red and yellow beetroot juices on blood pressure in free-living normotensive males: a pilot study
}

\author{
H.M. Cheng, H.K. Cheng and T.W. George \\ Department of Applied Sciences, Faculty of Health and Life Sciences, Northumbria University, Newcastle upon Tyne, \\ NE1 $8 S T$
}

Individuals with high blood pressure (BP) have a two to three-fold increase in the risk of developing cardiovascular disease when compared with healthy individuals ${ }^{(1)}$. High nitrate diets have been shown to lower $\mathrm{BP}^{(2)}$. Beetroot juice $(\mathrm{BJ})$ is rich in nitrate and has been shown to significantly reduce systolic BP (SBP) and diastolic BP (DBP) in normotensive individuals when given as a $250 \mathrm{~g}$ dose ${ }^{(3)}$. The peak reduction in SBP and DBP occurred at 2-3 hr. Beetroot also contains betalains which have been shown to act as antioxidants, inhibit lipid peroxidation and reduce ICAM1 expression in vitro ${ }^{(4,5)}$. Betalains are divided into two subclasses: betacyanins and betaxanthins, responsible for the colour of red and yellow beetroot respectively ${ }^{(6)}$. The present study investigated whether red and yellow BJ affects BP to the same extent and if betalains contribute an additional differential effect.

Seven normotensive males, aged 18-45 years, participated in a randomised, controlled, cross-over study investigating the effect of high nitrate juice (red or yellow beetroot) on BP compared to a low nitrate control (carrot juice). Following a 1 day low nitrate diet, fasted participants had an ambulatory blood pressure (ABP) monitor fitted. After baseline BP was recorded they consumed $250 \mathrm{~g}$ of juice and were then free to go about their normal daily activities. ABP was measured for $24 \mathrm{hr}$ post consumption. The participants consumed all three juices in a random order with at least one week washout period between treatments.

Overall, there was a significant difference in absolute change in SBP during the first 4 hours between treatments $(\mathrm{P}=0 \cdot 031)$, but no time by treatment effect (Fig. 1a). When comparing the area under the $\Delta$ SBP curves (Fig. 1b), there was a significant difference between carrot and yellow beetroot juices $(P=0.023)$, but no significant difference between red and yellow BJ. There was no significant difference between juices on DBP.
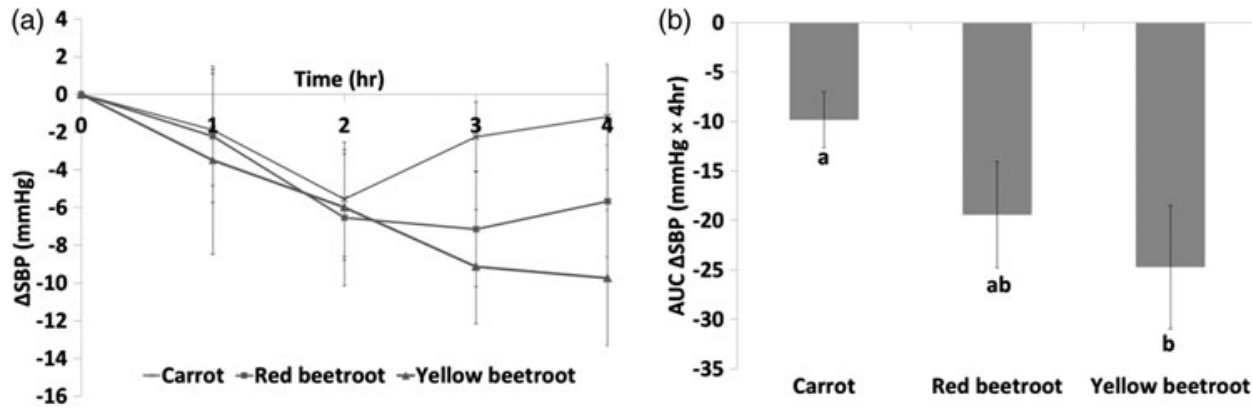

Fig. 1. (a) The mean absolute change of SBP in first $4 \mathrm{hr}$ following juice consumption $(\mathrm{n}=7)$. There was a significant treatment effect $(P=0.031)$, but no treatment by time effect. (b) The mean area under the curve of absolute change of SBP in first $4 \mathrm{hr}$ following juice consumption $(\mathrm{n}=7$ ). There was a significant difference between carrot and yellow beetroot juice $(\mathrm{P}=0.023)$.

Preliminary data suggests that yellow BJ decreased SBP more than carrot juice. Despite being lower in nitrate, carrot juice also reduced $\mathrm{BP}$ to the same extent as red BJ, which could be due it containing sufficient nitrate to exert a BP lowering effect. There was no significant difference between beetroot colour variants on SBP and DBP, suggesting there is no differential effect of betalains on BP.

1. Wang WY et al. (2006) Hypertension 47, 403-409.

2. Hobbs DA, TW George \& Lovegrove JA (2013) Nutrition Research Reviews 26, 210-222.

3. Hobbs DA et al. (2012) Br. J. Nutr 108, 2066-2074.

4. Halvorsen BL et al. (2002) J. Nutr 132, 461-471.

5. Kanner J, Harel S \& Granit R (2001) J. Agric. Food Chem 49, 5178-5185.

6. Kujala T, Loponen J \& Pihlaja K (2001) Z.Naturforsch. (C) 56, 343-348. 